\title{
On the mixed Dirichlet-Farwig biharmonic problem in exterior domains
}

\author{
Hovik Matevossian ${ }^{1,2}$ \\ ${ }^{1}$ Federal Research Center "Computer Science and Control", Russian Academy of Sciences, \\ Vavilov str., 40, Moscow 119333, Russia \\ ${ }^{2}$ Moscow Aviation Institute (National Research University), Volokolomskoe Shosse, 4, \\ Moscow 125993, Russia \\ E-mail: hmatevossian@graduate.org
}

\begin{abstract}
We study the properties of generalized solutions in unbounded domains and the asymptotic behavior of solutions of elliptic boundary value problems at infinity. Moreover, we study the unique solvability of the mixed Dirichlet-Farwig biharmonic problem in the exterior of a compact set under the assumption that generalized solutions of these problems has a bounded Dirichlet integral with weight $|x|^{a}$. Admitting different boundary conditions, we used the variation principle and depending on the value of the parameter $a$, we obtained uniqueness (non-uniqueness) theorems of the problem or present exact formulas for the dimension of the space of solutions.
\end{abstract}

Keywords: biharmonic operator; mixed Dirichlet-Farwig problem; Dirichlet integral; weighted spaces

MSC: 35J35; 35J40; 31B30

\section{Introduction}

Let $\Omega$ be an unbounded domain in $\mathbb{R}^{n}, n \geq 2, \Omega=\mathbb{R}^{n} \backslash \bar{G}$ with the boundary $\partial \Omega \in C^{2}$, where $G$ is a bounded simply connected domain (or a union of finitely many such domains) in $\mathbb{R}^{n}$, $0 \in G, \bar{\Omega}=\Omega \cup \partial \Omega$ is the closure of $\Omega, x=\left(x_{1}, \ldots, x_{n}\right) \in \mathbb{R}^{n}$ and $|x|=\sqrt{x_{1}^{2}+\cdots+x_{n}^{2}}$.

In $\Omega$ we consider the following problems for the biharmonic equation

$$
\Delta^{2} u=0
$$

with the mixed Dirichlet-Farwig boundary conditions

$$
\left.u\right|_{\Gamma_{1}}=\left.\frac{\partial u}{\partial \nu}\right|_{\Gamma_{1}}=0,\left.\quad \frac{\partial u}{\partial \nu}\right|_{\Gamma_{2}}=\left.\frac{\partial \Delta u}{\partial \nu}\right|_{\Gamma_{2}}=0
$$

where $\bar{\Gamma}_{1} \cup \bar{\Gamma}_{2}=\partial \Omega, \Gamma_{1} \cap \Gamma_{2}=\emptyset$ mes $_{n-1} \Gamma_{1} \neq 0, \nu=\left(\nu_{1}, \ldots, \nu_{n}\right)$ is the outer unit normal vector to $\partial \Omega$.

As is well known, if $\Omega$ is an unbounded domain, one should additionally characterize the behavior of the solution at infinity. As a rule, to this end, one usually poses either the condition that the Dirichlet (energy) integral is finite or a condition on the character of vanishing of the modulus of the solution as $|x| \rightarrow \infty$. Such conditions at infinity are natural and were studied by several authors (e.g., [11]- [13]). 
The behavior of solutions of the Dirichlet problem for the biharmonic equation as $|x| \rightarrow \infty$ was considered in [8], [9], where estimates for $|u(x)|$ and $|\nabla u(x)|$ as $|x| \rightarrow \infty$ were obtained under certain geometric conditions on the domain boundary.

Note that standard elliptic regularity results are available in [5]. The monograph covers higher order linear and nonlinear elliptic boundary value problems, mainly with the biharmonic or polyharmonic operator as leading principal part. The underlying models and, in particular, the role of different boundary conditions are explained in detail. As for linear problems, after a brief summary of the existence theory and $L^{p}$ and Schauder estimates, the focus is on positivity. The required kernel estimates are also presented in detail.

In [4], the boundary value problems for the biharmonic equation and the Stokes system are studied in a half space, and, using the Schwartz reflection principle in weighted $L^{q}$-space, the uniqueness of solutions of the Stokes system or the biharmonic equation is proved.

We also point out [1]- [3], in which using the methods of complex analysis the Dirichlet and Neumann problems for the polyharmonic equation are explicitly solved in the unit disc of the complex plane. The solution is obtained by modifying the related Cauchy-Pompeiu representation with the help of the polyharmonic Green function.

In the present note, this condition is the boundedness of the weighted Dirichlet integral:

$$
D_{a}(u, \Omega) \equiv \int_{\Omega}|x|^{a} \sum_{|\alpha|=2}\left|\partial^{\alpha} u\right|^{2} d x<\infty, \quad a \in \mathbb{R} .
$$

In various classes of unbounded domains with finite weighted Dirichlet (energy) integral, one of the author [14]- [24] studied uniqueness (non-uniqueness) problem and found the dimensions of the spaces of solutions of boundary value problems for the elasticity system and the biharmonic (polyharmonic) equation.

By developing an approach based on the use of Hardy type inequalities [7], [11]- [13], in the present note, we obtain a uniqueness (non-uniqueness) criterion for a solution of the mixed Dirichlet-Steklov-type and Steklov-type problems for the biharmonic equation.

Notation: $C_{0}^{\infty}(\Omega)$ is the space of infinitely differentiable functions in $\Omega$ with compact support in $\Omega$.

We denote by $H^{m}(\Omega, \Gamma), \Gamma \subset \bar{\Omega}$, the Sobolev space of functions in $\Omega$ obtained by the completion of $C^{\infty}(\bar{\Omega})$ vanishing in a neighborhood of $\Gamma$ with respect to the norm

$$
\left\|u ; H^{m}(\Omega, \Gamma)\right\|=\left(\int_{\Omega} \sum_{|\alpha| \leq m}\left|\partial^{\alpha} u\right|^{2} d x\right)^{1 / 2}, \quad m=1,2,
$$

where $\partial^{\alpha} \equiv \partial^{|\alpha|} / \partial x_{1}^{\alpha_{1}} \ldots \partial x_{n}^{\alpha_{n}}, \alpha=\left(\alpha_{1}, \ldots, \alpha_{n}\right)$ is a multi-index, $\alpha_{i} \geq 0$ are integers, and $|\alpha|=\alpha_{1}+\cdots+\alpha_{n}$; if $\Gamma=\emptyset$, we denote $H^{m}(\Omega, \Gamma)$ by $H^{m}(\Omega)$.

$H \quad(\Omega)$ is the space obtained by the completion of $C_{0}^{\infty}(\Omega)$ with respect to the norm $\left\|u(x) ; H^{m}(\Omega)\right\|$;

$\stackrel{\circ}{H}_{l o c}(\Omega)$ is the space obtained by the completion of $C_{0}^{\infty}(\Omega)$ with respect to the family of semi-norms

$$
\left\|u ; H^{m}\left(\Omega \cap B_{0}(R)\right)\right\|=\left(\int_{\Omega \cap B_{0}(R)} \sum_{|\alpha| \leq m}\left|\partial^{\alpha} u\right|^{2} d x\right)^{1 / 2}
$$

for all open balls $B_{0}(R):=\{x:|x|<R\}$ in $\mathbb{R}^{n}$ for which $\Omega \cap B_{0}(R) \neq \emptyset$.

Let $\left(\begin{array}{l}n \\ k\end{array}\right)$ be the $(n, k)$ - binomial coefficient, $\left(\begin{array}{l}n \\ k\end{array}\right)=0$ for $k>n$. 


\section{Definitions and auxiliary statements}

Definition 2.1. A solution of the homogenous biharmonic equation (1) in $\Omega$ is a function $u \in H_{\text {loc }}^{2}(\Omega)$ such that, for every function $\varphi \in C_{0}^{\infty}(\Omega)$, the following integral identity holds:

$$
\int_{\Omega} \Delta u \Delta \varphi d x=0
$$

Lemma 2.2. Let $u$ be a solution of equation (1) in $\Omega$ such that $D_{a}(u, \Omega)<\infty$. Then

$$
u(x)=P(x)+\sum_{\beta_{0}<|\alpha| \leq \beta} \partial^{\alpha} \Gamma(x) C_{\alpha}+u^{\beta}(x), \quad x \in \Omega,
$$

where $P(x) \quad$ is a polynomial, ord $P(x)<m_{0}=\max \{2,2-n / 2-a / 2\}, \beta_{0}=2-n / 2+a / 2$, $\Gamma(x)$ is the fundamental solution of equation (1), $C_{\alpha}=$ const, $\beta \geq 0$ is an integer, and the function $u^{\beta}$ satisfies the estimate:

$$
\left|\partial^{\gamma} u^{\beta}(x)\right| \leq C_{\gamma \beta}|x|^{3-n-\beta-|\gamma|}, C_{\gamma \beta}=\text { const },
$$

for every multi-index $\gamma$.

Remark 2.3. As is known [26], the fundamental solution $\Gamma(x)$ of the biharmonic equation has the form

$$
\Gamma(x)=\left\{\begin{array}{l}
C|x|^{4-n}, \text { if } 4-n<0 \text { or } n \text { is odd, } \\
C|x|^{4-n} \ln |x|, \text { if } 4-n \geq 0 \text { and } n \text { is even. }
\end{array}\right.
$$

Proof of Lemma 2.2 Consider the function $v(x)=\theta_{N}(x) u(x)$, where $\theta_{N}(x)=\theta(|x| / N), \theta \in$ $C^{\infty}\left(\mathbb{R}^{n}\right), 0 \leq \theta \leq 1, \theta(s)=0$ for $s \leq 1, \theta(s)=1$ for $s \geq 2$, while $N \gg 1$ and $G \subset\{x:|x|<N\}$. We extend $v$ to $\mathbb{R}^{n}$ by setting $v=0$ on $G=\mathbb{R}^{n} \backslash \bar{\Omega}$.

Then the function $v$ belongs to $C^{\infty}\left(\mathbb{R}^{n}\right)$ and satisfies the equation

$$
\Delta^{2} v=f,
$$

where $f \in C_{0}^{\infty}\left(\mathbb{R}^{n}\right)$ and $\operatorname{supp} f \subset\{x:|x|<2 N\}$. It is easy to see that $D_{a}\left(v, \mathbb{R}^{n}\right)<\infty$.

We can now use Theorem 1 of [10] since it is based on Lemma 2 of [10], which imposes no constraint on the sign of $\sigma$. Hence, the expansion

$$
v(x)=P(x)+\sum_{\beta_{0}<|\alpha| \leq \beta} \partial^{\alpha} \Gamma(x) C_{\alpha}+v^{\beta}(x),
$$

holds for each $a$, where $P(x)$ is a polynomial of order ord $P(x)<m_{0}=\max \{2,2-n / 2-a / 2\}$, $\beta_{0}=2-n / 2+a / 2, C_{\alpha}=$ const and

$$
\left|\partial^{\gamma} v^{\beta}(x)\right| \leq C_{\gamma \beta}|x|^{3-n-\beta-|\gamma|}, \quad C_{\gamma \beta}=\text { const } .
$$

Therefore, by the definition of $v$, we obtain (3). The proof of Lemma 2.2 is complete.

\section{Main Results}

Definition 3.1. By a solution of the mixed Dirichlet-Farwig problem (1), (2) we mean a function $u \underset{\stackrel{\circ}{H}_{l o c}^{2}}{l}\left(\Omega, \Gamma_{1}\right) \cap \stackrel{\circ}{H_{l o c}^{1}}(\Omega), \partial u / \partial \nu=0$ on $\Gamma_{2}$, such that, for every function $\varphi \in \stackrel{\circ}{H}_{l o c}^{2}\left(\Omega, \Gamma_{1}\right) \cap C_{0}^{\infty}\left(\mathbb{R}^{n}\right), \partial \varphi / \partial \nu=0$ on $\Gamma_{2}$, the following integral identity holds:

$$
\int_{\Omega} \Delta u \Delta \varphi d x=0
$$


Theorem 3.2. The mixed Dirichlet-Farwig problem (1),(2) with the condition $D(u, \Omega)<\infty$ has $n+1$ linearly independent solutions.

Proof. For any nonzero vector $A$ in $\mathbb{R}^{n}$, we construct a generalized solution $u_{A}$ of the biharmonic equation (1) with the boundary conditions

$$
\left.u_{A}(x)\right|_{\Gamma_{1}}=\left.(A x)\right|_{\Gamma_{1}},\left.\quad \frac{\partial u_{A}(x)}{\partial \nu}\right|_{\Gamma_{1}}=\left.\frac{\partial(A x)}{\partial \nu}\right|_{\Gamma_{1}},\left.\quad \frac{\partial u_{A}}{\partial \nu}\right|_{\Gamma_{2}}=\left.\frac{\partial \Delta u_{A}}{\partial \nu}\right|_{\Gamma_{2}}=0
$$

and the condition

$$
\chi\left(u_{A}, \Omega\right) \equiv\left\{\begin{array}{l}
\int_{\Omega}\left(\frac{\left|u_{A}\right|^{2}}{|x|^{4}}+\frac{\left|\nabla u_{A}\right|^{2}}{|x|^{2}}+\left|\nabla \nabla u_{A}\right|^{2}\right) d x<\infty \\
\int_{\Omega}\left(\frac{\left|u_{A}\right|^{2}}{\left.|| x\right|^{2} \ln |x|^{2}}+\frac{\left|\nabla u_{A}\right|^{2}}{\left.|| x|\ln | x\right|^{2}}+\left|\nabla \nabla u_{A}\right|^{2}\right) d x<\infty \\
\text { for } n>4, n \leq 4,
\end{array}\right.
$$

for $A, x \in \mathbb{R}^{n}$, where $A x$ denotes the standard scalar product of $A$ and $x$.

Such a solution of problem (1), (5) can be constructed by the variational method [26], minimizing the functional

$$
\Phi(v)=\frac{1}{2} \int_{\Omega}|\Delta v|^{2} d x
$$

in the class of admissible functions $\left\{v: v \in H^{2}(\Omega),\left.v(x)\right|_{\Gamma_{1}}=\left.(A x)\right|_{\Gamma_{1}},\left.\frac{\partial v(x)}{\partial \nu}\right|_{\Gamma_{1}}=\left.\frac{\partial(A x)}{\partial \nu}\right|_{\Gamma_{1}}\right.$, $\left.\frac{\partial v}{\partial \nu}\right|_{\Gamma_{2}}=\left.\frac{\partial \Delta v}{\partial \nu}\right|_{\Gamma_{2}}=0, v$ is compactly supported in $\left.\bar{\Omega}\right\}$.

The validity of condition (6) as a consequence of the Hardy inequality follows from the results in [11]- [13].

Now, for any arbitrary number $e \neq 0$, we construct a generalized solution $u_{e}$ of equation (1) with the boundary conditions

$$
\left.u_{e}\right|_{\Gamma_{1}}=e,\left.\quad \frac{\partial u_{e}}{\partial \nu}\right|_{\Gamma_{1}}=0,\left.\quad \frac{\partial u_{e}}{\partial \nu}\right|_{\Gamma_{2}}=\left.\frac{\partial \Delta u_{e}}{\partial \nu}\right|_{\Gamma_{2}}=0
$$

and the condition

$$
\chi\left(u_{e}, \Omega\right) \equiv\left\{\begin{array}{l}
\int_{\Omega}\left(\frac{\left|u_{e}\right|^{2}}{|x|^{4}}+\frac{\left|\nabla u_{e}\right|^{2}}{|x|^{2}}+\left|\nabla \nabla u_{e}\right|^{2}\right) d x<\infty \\
\int_{\Omega}\left(\frac{\left|u_{e}\right|^{2}}{\left.|| x\right|^{2} \ln |x|^{2}}+\frac{\left|\nabla u_{e}\right|^{2}}{\left.|| x|\ln | x\right|^{2}}+\left|\nabla \nabla u_{e}\right|^{2}\right) d x<\infty \\
\text { for } 2 \leq n \leq 4 .
\end{array}\right.
$$

The solution of problem (1), (7) also is constructed by the variational method with the minimization of the corresponding functional in the class of admissible functions $\{v: v \in$ $H^{2}(\Omega),\left.v\right|_{\Gamma_{1}}=e,\left.\frac{\partial v}{\partial \nu}\right|_{\Gamma_{1}}=0,\left.\frac{\partial v}{\partial \nu}\right|_{\Gamma_{2}}=\left.\frac{\partial \Delta v}{\partial \nu}\right|_{\Gamma_{2}}=0, \quad v$ is compactly supported in $\left.\bar{\Omega}\right\}$. [13].

The condition (8) as a consequence of the Hardy inequality follows from the results in [11]-

Consider the function $v=\left(u_{A}-A x\right)-\left(u_{e}-e\right)$. 
Obviously, $v$ is a solution of problem (1), (2):

$$
\Delta^{2} v=0, \quad x \in \Omega,\left.\quad v\right|_{\Gamma_{1}}=\left.\frac{\partial v}{\partial \nu}\right|_{\Gamma_{1}}=0,\left.\quad \frac{\partial v}{\partial \nu}\right|_{\Gamma_{2}}=\left.\frac{\partial \Delta v}{\partial \nu}\right|_{\Gamma_{2}}=0 .
$$

One can easily see that $v \not \equiv 0$ and $D(v, \Omega)<\infty$.

To each nonzero vector $\mathbf{A}=\left(A_{0}, A_{1}, \ldots, A_{n}\right)$ in $\mathbb{R}^{n+1}$, there corresponds a nonzero solution $v_{\mathbf{A}}=\left(v_{A_{0}}, v_{A_{1}}, \ldots, v_{A_{n}}\right)$ of problem (1), (2) with the condition $D\left(v_{\mathbf{A}}, \Omega\right)<\infty$, and moreover,

$$
v_{\mathbf{A}}=u_{A}-u_{e}-A x+e .
$$

Let $A_{0}, A_{1}, \ldots, A_{n}$ be a basis in $\mathbb{R}^{n+1}$. Let us prove that the corresponding solutions $v_{A_{0}}, v_{A_{1}}, \ldots, v_{A_{n}}$ are linearly independent. Let

$$
\sum_{i=0}^{n} C_{i} v_{A_{i}} \equiv 0, \quad C_{i}=\text { const } .
$$

Set $W \equiv \sum_{i=1}^{n} C_{i} A_{i} x-C_{0} e$. We have

$$
\begin{gathered}
W=\sum_{i=1}^{n} C_{i} u_{A_{i}}-C_{0} u_{e}, \\
\int_{\Omega}|x|^{-2}|\nabla W|^{2} d x<\infty, \quad n>4, \\
\left.\int_{\Omega}|| x|\ln | x\right|^{-2}|\nabla W|^{2} d x<\infty, \quad 2 \leq n \leq 4 .
\end{gathered}
$$

Let us show that

$$
W \equiv \sum_{i=1}^{n} C_{i} A_{i} x-C_{0} e \equiv 0 .
$$

Let $T=\sum_{i=0}^{n} C_{i} A_{i}=\left(t_{0}, \ldots, t_{n}\right)$, where $A_{0}=-e$. Then

$$
\begin{gathered}
\int_{\Omega}|x|^{-2}|\nabla W|^{2} d x=\int_{\Omega}|x|^{-2}\left(t_{1}^{2}+\cdots+t_{n}^{2}\right) d x=\infty, \quad n>4, \\
\int_{\Omega}|| x|\ln | x||^{-2}|\nabla W|^{2} d x=\left.\int_{\Omega}|| x|\ln | x\right|^{-2}\left(t_{1}^{2}+\cdots+t_{n}^{2}\right) d x=\infty, \quad 2 \leq n \leq 4,
\end{gathered}
$$

if $T \neq 0$.

Consequently, $T=\sum_{i=0}^{n} C_{i} A_{i}=0$, and since the vectors $A_{0}, A_{1}, \ldots, A_{n}$ are linearly independent, we obtain $C_{i}=0, i=0,1, \ldots, n$.

Thus, the Dirichlet-Farwig problem (1), (2) with the condition $D(u, \Omega)<\infty$ has at least $n+1$ linearly independent solutions.

Let us prove that each solution $u$ of problem (1), (2) with the condition $D(u, \Omega)<\infty$ can be represented as a linear combination of the functions $v_{A_{0}}, v_{A_{1}}, \ldots, v_{A_{n}}$, i.e.

$$
u=\sum_{i=0}^{n} C_{i} v_{A_{i}}, \quad C_{i}=\text { const } .
$$

Since $A_{0}, A_{1}, \ldots, A_{n}$ is a basis in $\mathbb{R}^{n+1}$, it follows that there exists constants $C_{0}, C_{1}, \ldots, C_{n}$ such that

$$
A=\sum_{i=0}^{n} C_{i} A_{i} .
$$


We set

$$
u_{0} \equiv u-\sum_{i=0}^{n} C_{i} v_{A_{i}} .
$$

Obviously, the function $u_{0}$ is a solution of problem $\chi\left(u_{0}, \Omega\right)<\infty$.

(1), (2), and $D\left(u_{0}, \Omega\right)<\infty$

Let us show that $u_{0} \equiv 0, x \in \Omega$. To this end, we substitute the function $\varphi(x)=u_{0}(x) \theta_{N}(x)$ into the integral identity (4) for the function $u_{0}$, where $\theta_{N}(x)=\theta(|x| / N), \theta \in C^{\infty}(\mathbb{R}), 0 \leq \theta \leq 1$, $\theta(s)=0$ for $s \geq 2$ and $\theta(s)=1$ for $s \leq 1$; then we obtain

$$
\int_{\Omega}\left(\Delta u_{0}\right)^{2} \theta_{N}(x) d x=-J_{1}\left(u_{0}\right)-J_{2}\left(u_{0}\right)
$$

where

$$
J_{1}\left(u_{0}\right)=2 \int_{\Omega} \Delta u_{0} \nabla u_{0} \nabla \theta_{N}(x) d x, \quad J_{2}\left(u_{0}\right)=\int_{\Omega} u_{0} \Delta u_{0} \Delta \theta_{N}(x) d x .
$$

By applying the Cauchy-Schwarz inequality and by taking into account the conditions $D\left(u_{0}, \Omega\right)<\infty$ and $\chi\left(u_{0}, \Omega\right)<\infty$, one can easily show that $J_{1}\left(u_{0}\right) \rightarrow 0$ and $J_{2}\left(u_{0}\right) \rightarrow 0$ as $N \rightarrow \infty$. Consequently, by passing to the limit as $N \rightarrow \infty$ in (9), we obtain

$$
\int_{\Omega}\left(\Delta u_{0}\right)^{2} d x=0
$$

Therefore, we have

$$
\begin{gathered}
\Delta u_{0}=0, \quad x \in \Omega, \\
\left.u_{0}\right|_{\Gamma_{1}}=\left.\frac{\partial u_{0}}{\partial \nu}\right|_{\Gamma_{1}}=0,\left.\quad \frac{\partial u_{0}}{\partial \nu}\right|_{\Gamma_{2}}=\left.\frac{\partial \Delta u_{0}}{\partial \nu}\right|_{\Gamma_{2}}=0 .
\end{gathered}
$$

Hence, it follows $\left[6\right.$, Ch.2] that $u_{0}=0$ in $\Omega$. The proof of the theorem is complete.

Theorem 3.3. The mixed Dirichlet-Farwig problem (1), (2) with the condition $D_{a}(u, \Omega)<\infty$ has:

(i) the trivial solution for $n-2 \leq a<\infty, n>4$;

(ii) $n$ linearly independent solutions for $n-4 \leq a<n-2, n>4$;

(iii) $n+1$ linearly independent solutions for $-n \leq a<n-4, n>4$;

(iv) $k(r, n)$ linearly independent solutions for $-2 r+2-n \leq a<-2 r+4-n, r>1, n>4$, where

$$
k(r, n)=\left(\begin{array}{c}
r+n \\
n
\end{array}\right)-\left(\begin{array}{c}
r+n-4 \\
n
\end{array}\right)
$$

The proof of Theorem 3.3 is based on Lemma 2.2 about the asymptotic expansion of the solution of the biharmonic equation and the Hardy type inequalities for unbounded domains [11]- [13]. In case $(i v)$, we need to determine the number of linearly independent solutions of the biharmonic equation (1), the degree of which not exceed the fixed number.

It is well know that the dimension of the space of all polynomials in $\mathbb{R}^{n}$ of degree $\leq r$ is equal $\left(\begin{array}{c}r+n \\ n\end{array}\right)$ [25]. Then the dimension of the space of all biharmonic polynomials in $\mathbb{R}^{n}$ of degree $\leq r$ is equal to

$$
\left(\begin{array}{c}
r+n \\
n
\end{array}\right)-\left(\begin{array}{c}
r+n-4 \\
n
\end{array}\right)
$$

since the biharmonic equation is the vanishing of some polynomial of degree $r-4$ in $\mathbb{R}^{n}$. If we denote by $k(r, n)$ the number of linearly independent polynomial solutions of equation (1) 
whose degree do not exceed $r$ and by $l(r, n)$ the number of linearly independent homogeneous polynomials of degree $r$, that are solutions of equation (1), then

$$
k(r, n)=\sum_{s=0}^{r} l(s, n),
$$

where

$$
l(s, n)=\left(\begin{array}{c}
s+n-1 \\
n-1
\end{array}\right)-\left(\begin{array}{c}
s+n-5 \\
n-1
\end{array}\right), \quad s>0 .
$$

Further, we prove that the mixed Dirichlet-Farwig problem (1), (2) with the condition $D_{a}(u, \Omega)<\infty$ for $-2 r+2-n \leq a<-2 r+4-n$ has equally $k(r, n)$ of linearly independent solutions.

\section{References}

[1] Begehr H 2005, Dirichlet problems for the biharmonic equation. General Math., 13, 65-72.

[2] Begehr H, Vu TNH and Zhang Z-X 2006, Polyharmonic Dirchlet problems. Proc. Steklov. Math. Inst., 255, 13-34.

[3] Begehr H, Vagenas CJ 2006, Iterated Neumann problem for the higher order Poisson equation. Math. Nachr., Bd.279, 38-57.

[4] Farwig R 1994, "A note on the reflection principle for the biharmonic equation and the Stokes system", Acta Appl. Math., 34 41-51.

[5] Gazzola F, Grunau H.-Ch and Sweers G 2010, Polyharmonic Boundary Value Problems: Positivity Preserving and Nonlinear Higher Order Elliptic Equations in Bounded Domains. Lecture Notes Math. 1991, SpringerVerlag.

[6] Gilbarg D and Trudinger N 1977, Elliptic Partial Differential Equations of Second Order. Berlin: SpringerVerlag.

[7] Egorov Yu V and Kondratiev V A 1996, On Spectral Theory of Elliptic Operators. Basel: Birkhauser.

[8] Kondratiev V A, Kopacek I and Oleinik O A 1981, "On asymptotic properties of solutions of the biharmonic equation", Diff. Equations, 17(10), 1886-1899.

[9] Kondratiev V A and Oleinik O A 1983, "Estimates for solutions of the Dirichlet problem for biharmonic equation in a neighbourhood of an irregular boundary point and in a neighbourhood of infinity SaintVenant's principle", Proc. Royal Soc. Edinburgh, 93A(3-4), 327-343.

[10] Kondratiev V A and Oleinik O A 1987, "On the behavior at infinity of solutions of elliptic systems with a finite energy integral", Arch. Rational Mech. Anal., 99(1), 75-99.

[11] Kondrat'ev V A and Oleinik O A 1988, "Boundary value problems for the system of elasticity theory in unbounded domains. Korn's inequalities", Russ. Math. Surveys, 43(5), 65-119.

[12] Kondratiev V A and Oleinik O A 1990, "Hardy's and Korn's Inequality and their Application", Rend. Mat. Appl., Serie VII. 10(3), 641-666.

[13] Kon'kov A A 1995, "On the dimension of the solution space of elliptic systems in unbounded domains", Russian Acad. Sci. Sbornik Math., 80(2), 411-434.

[14] Matevosyan O A 1998, "On solutions of boundary value problems for a system in the theory of elasticity and for the biharmonic equation in a half-space", Diff. Equations., 34(6), 803-808.

[15] Matevosyan O A 2001, "The exterior Dirichlet problem for the biharmonic equation: Solutions with bounded Dirichlet integral", Math. Notes, 70(3), 363-377.

[16] Matevossian H A 2003, "On solutions of mixed boundary-value problems for the elasticity system in unbounded domains", Izvestiya Math., 67(5), 895-929.

[17] Matevosyan O A 2014, "On solutions of a boundary value problem for a polyharmonic equation in unbounded domains", Russ. J. Math. Phys., 21(1), 130-132.

[18] Matevossian H A 2015, "On solutions of the Dirichlet problem for the polyharmonic equation in unbounded domains", P-Adic Numbers, Ultrametric Analysis, and Appl., 7(1), 74-78.

[19] Matevossian O A 2015, "On solutions of the Neumann problem for the biharmonic equation in unbounded domains", Math. Notes, 98, 990-994.

[20] Matevosyan O A 2016, "On solutions of the mixed Dirichlet-Navier problem for the polyharmonic equation in exterior domains", Russ. J. Math. Phys., 23(1), 135-138.

[21] Matevosyan O A 2016, "On solutions of one boundary value problem for the biharmonic equation", Diff. Equations, 52(10), 1379-1383. 
[22] Matevossian H A 2017, "On the biharmonic Steklov problem in weighted spaces", Russ. J. Math. Phys., 24(1), 134-138.

[23] Matevossian H A 2018, "On the Steklov-type biharmonic problem in unbounded domains", Russ. J. Math. Phys., 25:2, 271-276.

[24] Hovik A Matevossian 2019, "On the polyharmonic Neumann problem in weighted spaces", Complex Variables 86 Elliptic Equations, 64:1, 1-7.

[25] Mikhlin S G 1977, Linear Partial Differential Equations, Vyssaya Shkola, Moscow (in Russian).

[26] Sobolev S L 1988, Some Applications of Functional Analysis in Mathematical Physics, 3th ed., Nauka, Moscow; Applications of Functional Analysis in Mathematical Physics. Amer. Math. Soc., Providence 1991. 